\title{
Mycobacterial laminin-binding histone-like protein mediates collagen-dependent cytoadherence
}

\author{
André Alves Dias', Dominique Raze ${ }^{2,3,4,5}$, Cristiana Soares de Lima', \\ Maria Angela de Melo Marques ${ }^{6}$, Hervé Drobecq ${ }^{7}$, Anne-Sophie Debrie ${ }^{2,3,4,5}$, \\ Michelle Lopes Ribeiro-Guimarães ${ }^{1}$, Franck Biet ${ }^{8}$, Maria Cristina Vidal Pessolani ${ }^{1 /+}$
}

\begin{abstract}
${ }^{1}$ Laboratório de Microbiologia Celular, Instituto Oswaldo Cruz-Fiocruz, Rio de Janeiro, RJ, Brasil ²Inserm, U 1019, Lille, France ${ }^{3}$ CNRS, UMR $8204{ }^{7}$ CNRS, UMR 8525, Lille, France ${ }^{4}$ Institut Pasteur de Lille, Centre for Infection and Immunity of Lille (CIIL), Lille, France ${ }^{5}$ Université Lille Nord de France, Lille, France ${ }^{6}$ Department of Microbiology, Immunology and Patology, Colorado State University, Fort Collins, USA ${ }^{8}$ INRA, UR1282, Infectiologie et Santé Publique, Centre de Tours, Nouzilly, France
\end{abstract}

\begin{abstract}
When grown in the presence of exogenous collagen I, Mycobacterium bovis BCG was shown to form clumps. Scanning electron microscopy examination of these clumps revealed the presence of collagen fibres cross-linking the bacilli. Since collagen is a major constituent of the eukaryotic extracellular matrices, we assayed BCG cytoadherence in the presence of exogenous collagen I. Collagen increased the interaction of the bacilli with A549 type II pneumocytes or U937 macrophages, suggesting that BCG is able to recruit collagen to facilitate its attachment to host cells. Using an affinity chromatography approach, we have isolated a BCG collagen-binding protein corresponding to the previously described mycobacterial laminin-binding histone-like protein (LBP/Hlp), a highly conserved protein associated with the mycobacterial cell wall. Moreover, Mycobacterium leprae LBP/Hlp, a wellcharacterized adhesin, was also able to bind collagen I. Finally, using recombinant fragments of $\mathrm{M}$. leprae LBP/ Hlp, we mapped the collagen-binding activity within the C-terminal domain of the adhesin. Since this protein was already shown to be involved in the recognition of laminin and heparan sulphate-containing proteoglycans, the present observations reinforce the adhesive activities of LBP/Hlp, which can be therefore considered as a multifaceted mycobacterial adhesin, playing an important role in both leprosy and tuberculosis pathogenesis.
\end{abstract}

Key words: mycobacteria - cytoadherence - collagen - histone-like protein - leprosy - tuberculosis

Despite decades of investigations, tuberculosis and leprosy caused by Mycobacterium tuberculosis and Mycobacterium leprae, respectively, still represent major threats to human health. If $M$. leprae is a major concern in developing countries with an incidence of about 230.000 new cases per year (WHO 2010a), M. tuberculosis remains the world's leading cause of death due to a single infectious agent with 1.5 million deaths annually (WHO 2010b). Although these two bacterial species display different tropisms for human tissues, they both trigger infections characterized by a systemic dissemination step which requires specific interactions of the pathogen with host cell (Pessolani et al. 2003). The mechanisms responsible for the systemic mycobacterial dissemination remain poorly understood and their deciphering constitutes a major challenge since it may lead to the development of new prophylactic and/or therapeutic strategies. To better understand the molecular interplay between mycobacteria and host cells, we have initiated investigations dealing with the identification and characteriza-

Financial support: FIOCRUZ/PAPES III, INSERM, Institut Pasteur de Lille

+ Corresponding author: cpessola@ioc.fiocruz.br

Received 31 March 2012

Accepted 17 July 2012 tion of mycobacterial adhesins that interact with human host cells. These studies led us to identify a $22-\mathrm{kDa}$ surface-exposed heparin-binding haemagglutinin adhesin (HBHA) which is involved in the interaction of $M$. tuberculosis and M. leprae with epithelial cells, but not with professional phagocytes (Menozzi et al. 2006, Lima et al. 2009). In addition, HBHA which binds heparan sulphatecontaining receptors through its $\mathrm{C}$-terminal lysine-rich domain (Delogu \& Brennan 1999, Pethe et al. 2000) has been shown to be required for extrapulmonary dissemination (Pethe et al. 2001, Mueller-Ortiz et al. 2002). Recently, HBHA was shown to trigger receptor-mediated transcytosis, suggesting that mycobacteria may disseminate via a direct passage through an epithelial barrier (Menozzi et al. 2006). Besides HBHA, the histone-like protein (Hlp) has also been implicated in the attachment of pathogenic mycobacteria to host cells. Mycobacterial Hlp, a positively-charged, surface-exposed molecule with roughly twice the size of other bacterial Hlps, is a highly conserved protein shared by all mycobacterial species (Lefrançois et al. 2011). This protein was initially described as a laminin-binding protein (LBP) involved in M. leprae-Schwann cell (SC) interaction (Shimoji et al. 1999, Marques et al. 2000). More recently LBP/Hlp has been shown to also play a major role in mediating the adhesion of mycobacteria to epithelial respiratory cells by interacting with proteoglycan-containing receptors such as heparan sulphate and hyaluronic acid (HA) (Aoki et al. 2004, Lefrançois et al. 2011). 
Because collagenous proteins are the major constituents of the extracellular matrices of epithelial cells and may serve as receptors for bacterial adherence (Kreis \& Vale 1993), we have conducted experiments to know whether mycobacteria may directly interact with collagen. In the present study, we show that Mycobacterium bovis BCG grown in the presence of exogenous collagen I forms clusters. In addition, cytoadherence assays have revealed that the interaction of $M$. bovis BCG with pneumocytes and macrophages is increased in the presence of exogenous collagen or following a pretreatment of the mycobacteria with collagen. These observations indicated that $M$. bovis $\mathrm{BCG}$ is able to recruit collagen, suggesting the presence of a surface-exposed collagen-binding receptor. This receptor has been purified by affinity chromatography and demonstrated to be the LBP/Hlp protein. Finally, we mapped the collagen-binding activity of LBP/ Hlp within the C-terminal domain of the protein.

\section{MATERIALS AND METHODS}

Mycobacterial strains and growth conditions - M. bovis BCG (strain 1173P2, World Health Organization, Stockholm, Sweden) was grown at $37^{\circ} \mathrm{C}$ in tissue culture grade Roux flasks (Nunc) using Sauton medium supplemented or not with type 1 calf skin collagen (Collagen S, Roche, Mannheim, Germany) at the concentration of $25 \mu \mathrm{g} / \mathrm{mL}$. Mycobacterium smegmatis wild-type (wt) $\mathrm{mc}^{2} 155$ and the mutant for the $h l p$ gene $(\Delta h l p)$ were kindly provided by Thomas Dick of Singapore University, Singapore. The mutant was generated as previously described by Lee et al. (1998). Both of the strains were grown in Middlebrook 7H9 broth (Difco, Detroit, MI, USA) supplemented with $10 \%$ albumin, dextrose and $\mathrm{NaCl}$ (ADC) and $0.05 \%$ Tween- 80 and $0.5 \%$ glycerol under agitation. Mycobacteria were acid-fast stained by the Kinyoun method and visualised under light microscope.

Mycobacterial adherence assays - Exponentiallygrowing $M$. bovis $\mathrm{BCG}\left(\mathrm{OD}_{600}\right.$ of 0.4$)$ were collected by low-speed centrifugation $\left(6.500 \mathrm{~g}\right.$ for $10 \mathrm{~min}$ at $\left.4^{\circ} \mathrm{C}\right)$ and carefully resuspended in $50 \mathrm{mM}$ Tris- $\mathrm{HCl}(\mathrm{pH} 7.3)$ containing $150 \mathrm{mM} \mathrm{NaCl}$ [Tris buffered saline (TBS)]. The mycobacterial cytoadherence was then assayed as described previously (Menozzi et al. 1996) using A549 cells (human type II pneumocytes; ATCC, CCL 185) or U937 cells (human macrophages; ATCC, CCL 1593) grown in 24-well tissue culture trays. The adherence assays were performed at a multiplicity of infection of 10 in the absence or the presence of increasing concentrations of type 1 collagen. Following $2 \mathrm{~h}$ of incubation at $37^{\circ} \mathrm{C}$, the cells were washed three times with TBS, lysed by adding $1 \mathrm{~mL}$ distilled water containing $0.05 \%$ (wt/vol) sodium deoxycholate and serial dilutions were plated onto 7H11 medium for colony forming units (CFUs) counting. $\mathrm{Cy}-$ toadherence is expressed as the percentage of CFUs present in the inoculum that remain associated with target cells after the washing step. To investigate the effect of trypsin or collagen pretreatment on the $M$. bovis $\mathrm{BCG}$ cytoadherence, the bacteria were incubated for $30 \mathrm{~min}$. at $37^{\circ} \mathrm{C}$ in TBS supplemented with porcine pancreas trypsin (Sigma) or collagen I, respectively. After three washes with TBS, the bacilli were passed five times through a 28 -gauge needle to unravel possible clumps and finally submitted to the adherence assay as described above.

Affinity chromatography on collagen-Sepharose - Collagen I was covalently linked to CNBr-activated Sepharose 4B (Amersham) according to the manufacturer's recommendations. Briefly, $5 \mathrm{~mL}$ of gel swollen in distilled water was washed with $20 \mathrm{~mL} 1 \mathrm{mM} \mathrm{HCl}$ followed by $200 \mathrm{~mL}$ phosphate buffered saline (PBS) (10 mM phosphate buffer $\mathrm{pH} 7.2,0.15 \mathrm{M} \mathrm{NaCl}$ ). The gel was then resuspended in $25 \mathrm{~mL}$ PBS containing collagen $\mathrm{I}$ at the concentration of $1 \mathrm{mg} / \mathrm{mL}$ and incubated overnight at $4^{\circ} \mathrm{C}$ under gentle agitation. The remaining active groups were blocked by washing the gel with $200 \mathrm{~mL} 100 \mathrm{mM}$ Tris- $\mathrm{HCl} \mathrm{pH}$ 8.0. The collagen coupling efficiency ranged between $95-99 \%$. Fractions containing soluble extracts of $M$. bovis BCG were prepared as described previously (Menozzi et al. 1996) and chromatographed at a flow rate of $1.5 \mathrm{~mL} / \mathrm{min}$ over $5 \mathrm{~mL}$ of collagen-Sepharose matrix packed in a glass column (1-cm diameter) and equilibrated with PBS. At the end of the sample loading step, the gel was washed with $100 \mathrm{~mL}$ PBS and the bound material was eluted by a $0-1 \mathrm{M} \mathrm{NaCl}$ linear gradient in $100 \mathrm{~mL}$ PBS. Fractions of $1 \mathrm{~mL}$ were collected and their protein concentrations were determined by using the bicinchoninic acid method (Pierce) and bovine serum albumin (BSA) as a standard.

Protein identification - The protein band corresponding to the $M$. bovis BCG collagen-binding protein was excised from the Coomassie-stained polyacrylamide gel, washed twice with $50 \%$ acetonitrile prepared in $20 \mathrm{mM}$ ammonium hydrogenocarbonate and finally digested overnight within the gel fragment using $50 \mathrm{ng}$ trypsin (Promega, Madison, WI, USA). The resulting peptides were eluted, desalted with a ZIPTIP C18 column (Millipore, Billerica, MA) and spotted on a Maldi plate with $0.5 \mu \mathrm{L}$ of freshly dissolved $\alpha$-cyano-4-hydroxycinnaminic acid at $5 \mathrm{mg} / \mathrm{mL}$ in $50 \%$ acetonitrile and $0.1 \%$ trifluoroacetic acid. After drying, the spots were washed with $3 \mu \mathrm{L}$ of $20 \mathrm{mM}$ diammonium citrate $\mathrm{pH} 4.5$ and mass spectrometry analyses were performed by using a matrix-assisted laser desorption ionization/time-of-flight VoyagerDE-STR (Applied Biosystems, Palo Alto, CA). Peptides were analyzed by using the following setting parameters: positive and reflector modes, acceleration voltage of $20 \mathrm{kV}$, grid voltage of $61 \%, 90 \mathrm{~ns}$ of delayed extraction time and low mass gate $500 \mathrm{Da}$. The spectra were calibrated externally by using the $\left[\mathrm{M}+\mathrm{H}^{+}\right]$monoisotopic ions of peptides resulting from trypsin-digested lyzozyme. Database search based on the peptide masses observed was performed against the NCBInr database using the MS-FIT algorithm (Protein Prospector; 128.40.158.151/ mshome3.4.htm) or the ProFound algorithm (prowl.rockefeller.edu/profound_bin/WebProFound.exe).

Sodium dodecyl sulphate polyacrylamide gel electrophoresis (SDS-PAGE) and immunoblotting analysis - SDS-PAGE was performed using a 12-15\% gel and proteins were stained with Coomassie brilliant blue R-250 (ICN) or silver (Sigma). Alternately, proteins were trans- 
ferred onto nitrocellulose membranes (Protran BA85, Schleicher \& Schuell) as described. Immobilized proteins were probed with the 5G9 anti-rLBP/Hlp monoclonal antibody (1:1000) (Marques et al. 2000). The membranes were then washed three times with TBS/T and incubated with a goat anti-mouse alkaline phosphateconjugated antibody. The substrates nitro blue tetrazolium and 5-bromo-4-chloro-3-indolyl phosphate were used for colour development.

Scanning electron microscopy (SEM) - M. bovis BCG grown in Sauton medium to mid log phase in the absence or the presence of $25 \mu \mathrm{g} / \mathrm{mL}$ collagen I were resuspended in PBS $\left(\mathrm{OD}_{600}\right.$ of $\left.\sim 1.0\right)$. The bacteria were then fixed for $5 \mathrm{~h}$ at room temperature (RT) in a $1.25 \%(\mathrm{v} / \mathrm{v})$ glutaraldehyde solution prepared in $100 \mathrm{mM}$ sodium cacodylate buffer ( $\mathrm{pH}$ 7.0). A similar fixation step was applied to a collagen I solution at $50 \mu \mathrm{g} / \mathrm{mL}$ prepared in PBS. Next, the samples were filtered through $25 \mathrm{~mm}$ diameter/ 0.2 $\mu \mathrm{m}$ porosity Anodisc (Whatman) and the filters were rinsed five times for $10 \mathrm{~min}$ in $25 \mathrm{~mL}$ cacodylate buffer. Post-fixation was performed for $3 \mathrm{~h}$ in $1 \% \mathrm{OsO}_{4}$ solution prepared in cacodylate buffer, followed by five washes in ultrapure water. The samples underwent progressive dehydration by successive soaking in $50 \%, 70 \%, 95 \%$ and $100 \%$ ethanol. Soaking in isopentyl acetate was performed before critical point drying in $\mathrm{CO}_{2}$, using an EMSDCOPE CPD 750 apparatus. The filters were then attached to large SEM stubs and coated with gold/palladium by cathodic spreading in a Polaron E5100 coater. Sample observation and microphotographs were done in a JEOL JSM35CF scanning electron microscope, operating at a voltage of $10 \mathrm{kV}$.

Preparation of recombinant full-length $\mathrm{LBP} / \mathrm{Hlp}$ and truncated forms - The recombinant $M$. bovis BCG LBP/Hlp protein was obtained using the same procedure previously described for cloning and expression of the Mycobacterium avium subsp. paratuberculosis LBP/Hlp-encoding gene (Lefrançois et al. 2011). The M. bovis LBP/Hlp-encoding gene (GenBank accession GQ259334) was amplified by polymerase chain reaction from chromosomal DNA of $M$. bovis BCG strain $1173 \mathrm{P} 2$ using the $P f u$ DNA polymerase (Promega) and two synthetic oligonucleotides (Sigma) with the following sequences: 5'TATACATATGCACCACCACCA CCACCACATGAACAAAGCAGAGCTCATTGACG -3' (BCG-LBP/Hlp S) and 5'-TATAGCGGCCGCCTATTTGCGACCCCGCCGAGCGG-3' (BCG-LBP/Hlp AS), containing an NdeI and NotI sites, respectively. The oligonucleotide BCG-LBP/Hlp S sequence was designed to produce a hybrid protein carrying a His-tag at the Nterminal position used for affinity purification of the recombinant protein. Recombinant full-length $M$. leprae $\mathrm{LBP} / \mathrm{Hlp}(\mathrm{rLBP} / \mathrm{Hlp})$ and truncated forms of the protein corresponding to the N-terminal (residues 1-110; rLBP/ Hlp-N) and the C-terminal (residues 111-200; rLBP/ Hlp-C) were obtained as previously described (Marques et al. 2000, Lima et al. 2005).

Binding assays in microplates of recombinant $B C G$ $L B P / H l p$ to collagen $I$ - To investigate the capacity of $M$. bovis BCG rLBP/Hlp to bind to collagen, $1.0 \mu \mathrm{g} / \mathrm{mL}$ of collagen I (Sigma, St. Louis, MO) in $50 \mu \mathrm{L} 0.1 \mathrm{M}$ carbonate-bicarbonate buffer ( $\mathrm{pH}$ 9.6) was used to coat the wells of a polystyrene microplate (Corning, New York, NY). The microtitre plate was incubated overnight at $4^{\circ} \mathrm{C}$. The wells were then washed with PBS and blocked for $2 \mathrm{~h}$ with $200 \mu \mathrm{L}$ PBS-3\% BSA at RT. After washing with PBS $/ 0.05 \%$ Tween 20 (PBS/T), $50 \mu \mathrm{L}$ of increasing concentrations of $M$. bovis $\mathrm{BCG}$ rLBP/Hlp were added to the wells and incubated at $37^{\circ} \mathrm{C}$ for $2 \mathrm{~h}$. The wells were then rinsed with $\mathrm{PBS} / \mathrm{T}$ and incubated with the anti-LBP/Hlp monoclonal antibody 5G9 (1:500) for $1 \mathrm{~h}$ at $37^{\circ} \mathrm{C}$. After washing with $\mathrm{PBS} / \mathrm{T}$, rabbit anti-mouse $\mathrm{IgG}$ peroxidase conjugate (Sigma; 1:1000) was added and incubated for an additional $50 \mathrm{~min}$ at $37^{\circ} \mathrm{C}$. Peroxidase activity was revealed with hydrogen peroxide and $o$-phenylenediamine (OPD). The reaction was stopped with $\mathrm{HCl}$ and read at $490 \mathrm{~nm}$ with a TitertekPlus microplate reader (ICN Biomedicals Inc, Costa Mesa, CA). Control wells coated with BSA were included in all binding assays.

Mapping the collagen-binding site of M. leprae LBP/ $H l p$ - To monitor the binding of different types of soluble collagens to $M$. leprae rLBP/Hlp or truncated forms, $5 \mu \mathrm{g} / \mathrm{mL}$ of each protein in $0.1 \mathrm{M}$ carbonate buffer $\mathrm{pH}$ 9.6 $(50 \mu \mathrm{L})$ were used to coat the wells of polystyrene microplates (Corning, New York, NY). Plates were incubated overnight at $4^{\circ} \mathrm{C}$. The wells were then washed with PBS and blocked for $2 \mathrm{~h}$ at RT with $200 \mu \mathrm{L}$ PBS containing $2 \%$ BSA. Upon washing with PBS/T, $50 \mu \mathrm{L}$ of increasing concentrations of biotinylated collagens I, III, IV or VI (Sigma; $0-80 \mu \mathrm{g} / \mathrm{mL}$ ) were added to the wells and incubation was performed at RT for $2 \mathrm{~h}$. The wells were rinsed with PBS/T and incubated with streptavidin-peroxidase (Pierce, Rockford, IL) at $0.5 \mu \mathrm{g} / \mathrm{mL}$. Peroxidase activity was revealed with hydrogen peroxide and OPD. The reaction was stopped with $\mathrm{HCl}$ and read at $490 \mathrm{~nm}$ in a TitertekPlus microplate reader. In control wells, collagen was omitted and specific collagen-binding activity was determined by subtracting the absorbency resulting from non-specific binding detected in the control wells.

\section{RESULTS}

Collagen-induced agglutination of $M$. bovis $B C G$ - To investigate the interaction of $M$. bovis BCG with collagen, we first cultivated the mycobacteria in Sauton medium in the absence or the presence of collagen I at the concentration of $25 \mu \mathrm{g} / \mathrm{mL}$. In the control culture, the mycobacteria formed a thin layer that stuck loosely to the bottom of the Roux flask and the cells were easily resuspended by repeated shakings of the flask. When collagen I was added to the culture medium, the bacterial layer was more firmly bound to the flask surface and required the use of a cell scraper to completely detach the cells. In addition, once in suspension the mycobacteria were not evenly dispersed, but most of them remained agglutinated in small clumps. To examine the structure of these clumps, mycobacteria were observed by SEM. As shown in Fig. 1A and Supplementary data, M. bovis $\mathrm{BCG}$ cells grown in the absence of collagen failed to form aggregates and most of the bacteria appeared isolated on the filter membrane. In contrast, large bacterial aggregates were observed using $M$. bovis $\mathrm{BCG}$ from 
cultures performed in the presence of collagen I (Fig. 1B, Supplementary data). An amorphous and fibrillar material in contact with the bacteria was also observed within these aggregates. Because this material could represent collagen fibres cross-linked by glutaraldehyde during the sample preparation, collagen I was fixed and observed in the same conditions. Collagen appeared as long and entangled filaments exhibiting various thicknesses (Supplementary data). Since this aspect was quite similar to that of the extracellular material detected within the mycobacterial clumps, it suggests that $M$. bovis BCG may interact directly with collagen fibres.

Exogenous collagen I increases the cytoadherence of $M$. bovis $B C G$ - Because collagen is a predominant constituent of the extracellular matrices of epithelial cells (Kreis \& Vale 1993), we assayed the cytoadherence of $M$. bovis $\mathrm{BCG}$ in the presence of increasing collagen I concentrations ranging from $0-100 \mu \mathrm{g} / \mathrm{mL}$. These assays were performed using human type II A549 pneumocytes, but also U937 human macrophages since mycobacteria display a tropism for monocyte-derived phagocytic cells (Stokes et al. 1993, Ernst 1998). As shown in Fig. 2, a dose-dependent effect was observed for both cell lines. Compared with the control, a ca. three-fold increase in adherence was observed with the A549 pneumocytes in the presence of $40 \mu \mathrm{g} / \mathrm{mL}$ collagen I. The use of higher collagen concentrations did not further increase the mycobacterial adherence, indicating a saturable mechanism. The effect of collagen I on the interaction of $M$. bovis BCG with U937 macrophages was also shown
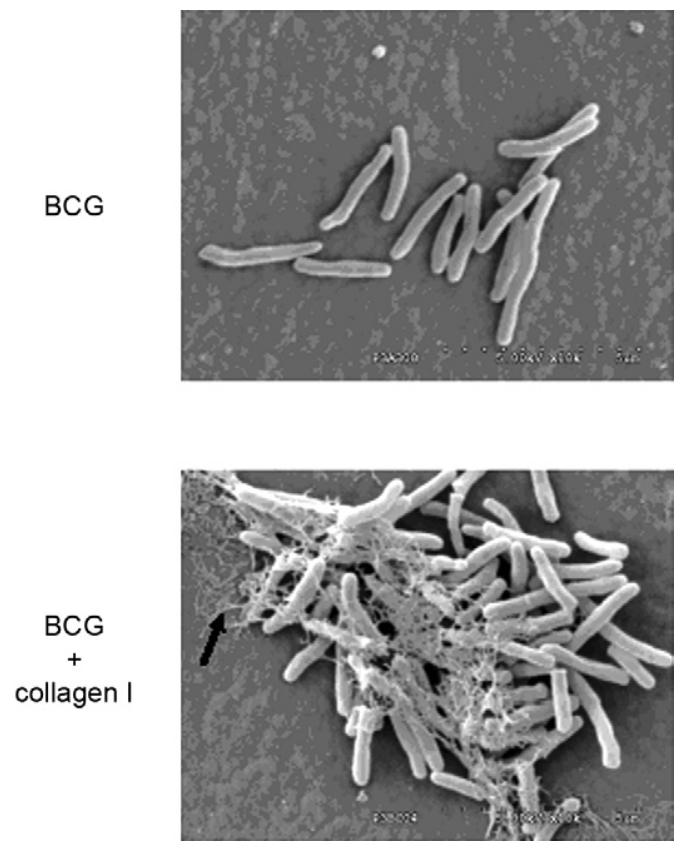

Fig. 1: Mycobacterium bovis BCG grown in the absence or the presence of collagen I. Bacilli grown in the absence (A) or the presence (B) of $25 \mu \mathrm{g} / \mathrm{mL}$ collagen I were fixed and observed by scanning electron microscopy. The arrow indicates collagen fibres that surround mycobacteria. to be saturable, but it appeared more pronounced since a four-fold increase in adherence was observed in the presence of $20 \mu \mathrm{g} / \mathrm{mL}$ collagen I.

Pretreatment of $M$. bovis BCG with collagen I increases its cytoadherence - In order to investigate the molecular mechanism leading to the increased cytoadherence of $M$. bovis BCG in the presence of exogenous collagen I, the bacilli were incubated in TBS containing $50 \mu \mathrm{g} / \mathrm{mL}$ collagen I prior to the cytoadherence assay. At the end of $30 \mathrm{~min}$ incubation at $37^{\circ} \mathrm{C}$, the mycobacteria were washed twice with TBS and then assayed for their capability to adhere to A549 pneumocytes and U937 macrophages. As shown in Fig. 3, such a collagen I pretreatment induced a ca. three-fold increase in the mycobacterial adherence onto both A549 pneumocytes and U937 macrophages. This observation suggests that $M$. bovis BCG may recruit exogenous collagen which may in turn favour the attachment of the bacilli to the eukaryotic cell surface. Such a recruitment was confirmed by the SDS-PAGE analysis of $M$. bovis BCG pretreated with collagen I followed by three washing steps with TBS. Indeed, the Coomassie blue staining of the gel revealed that collagen was present in the whole-cell lysate derived from mycobacteria pretreated with collagen (data not shown). This finding indicates that collagen I binds compounds probably associated with the $M$. bovis BCG surface. Therefore, these compounds could act as adhesins through their collagen-binding activity.

A proteinaceous $M$. bovis BCG compound is required for the collagen-dependent adherence - The recruitment of collagen by $M$. bovis BCG led us to characterize the mycobacterial surface compounds involved in this activ-

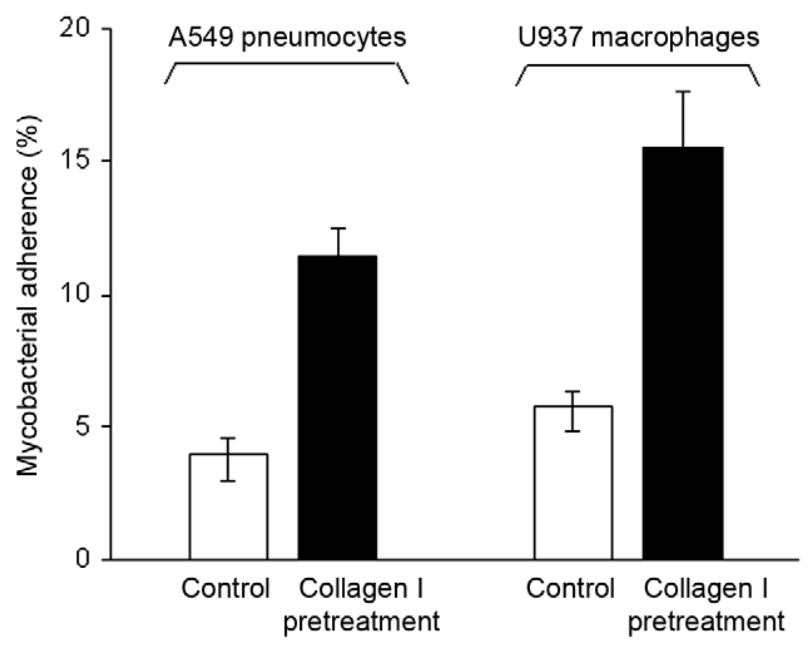

Fig. 2: effect of exogenous collagen I on the Mycobacterium bovis BCG interaction with pneumocytes and macrophages. Exponentiallygrowing bacilli were incubated for $2 \mathrm{~h}$ with A549 type II human pneumocytes or with human U937 macrophages using a multiplicity of infection of 10 in the presence of increasing concentrations of collagen I. After washing to remove unbound mycobacteria, colony forming units associated with both cell lines were counted by plating serial cell lysate dilutions onto 7H11 agar. The data represent averages for quadruplicate experiments and standard deviation bars are shown. 
ity. We first assayed the cytoadherence of mycobacteria pretreated for $30 \mathrm{~min}$ with porcine pancreas trypsin at the concentrations of 0.1 or $1 \mu \mathrm{g} / \mathrm{mL}$. These conditions were chosen because neither modified the viability of $M$. bovis BCG, as observed by CFUs determination following the trypsin pretreatment (data not shown). Pretreatment of the bacilli with trypsin at $1 \mu \mathrm{g} / \mathrm{mL}$ did not significantly modify the mycobacterial interaction with U937 macrophages, suggesting that this interaction does not mainly involve proteinaceous adhesins (Supplementary data). In contrast, such a trypsin pretreatment induced a ca. $60 \%$ reduction of the M. bovis BCG attachment to the A549 pneumocytes. This finding is in agreement with the fact that HBHA, which is the major epithelial adhesin produced by both M. bovis BCG and M. tuberculosis, exhibits a surface-exposed heparin-binding adhesive domain highly sensitive to proteolytic degradation (Pethe et al. 2002, Dupres et al. 2005). When bacilli pretreated with $0.1 \mu \mathrm{g} / \mathrm{mL}$ trypsin were used in the cytoadherence assay run in the presence of $50 \mu \mathrm{g} / \mathrm{mL}$ exogenous collagen I, the increase in myco-
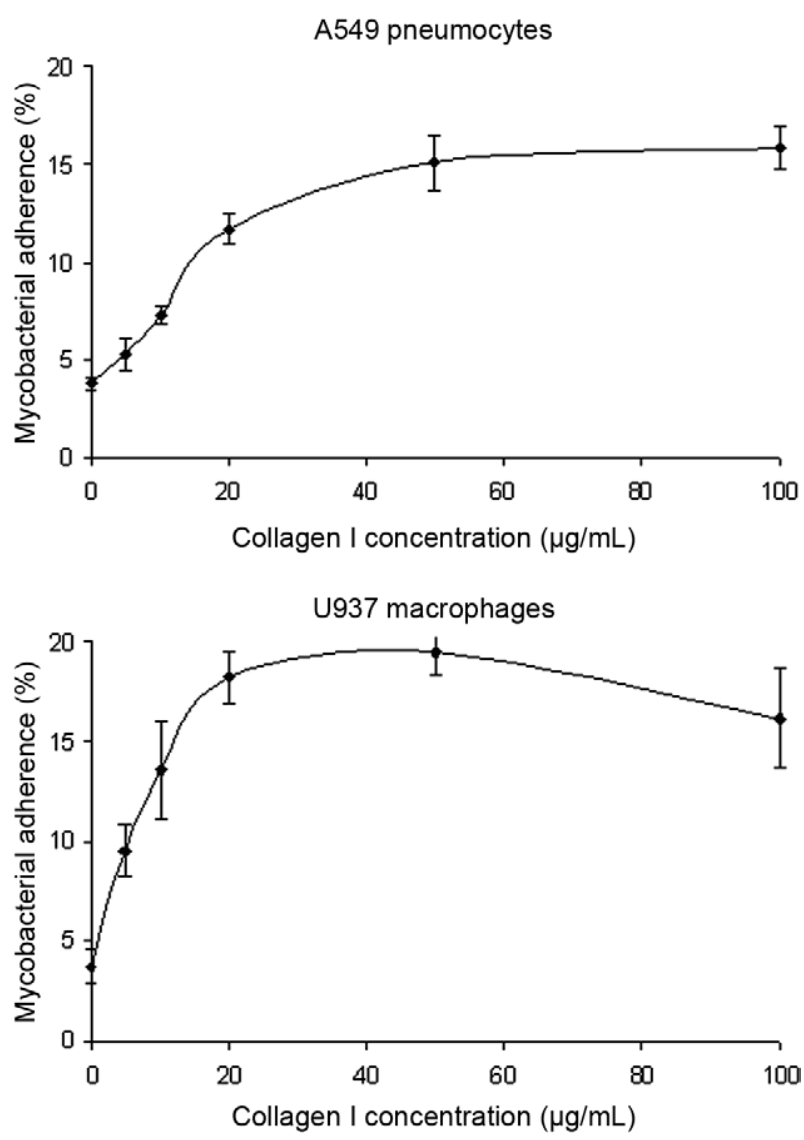

Fig. 3: effect of collagen pretreatment on the Mycobacterium bovis $\mathrm{BCG}$ cytoadherence. Exponentially-growing M. bovis BCG were pretreated or not with $50 \mu \mathrm{g} / \mathrm{mL}$ collagen I and then assayed for their interaction with A549 pneumocytes or U937 macrophages using a multiplicity of infection of 10 . Colony forming units associated with target cells were counted by plating serial cell lysate dilutions on $7 \mathrm{H} 11$ agar. The data with standard deviations represent averages for quadruplicate experiments. bacterial adherence to both A549 pneumocytes and U937 macrophages was reduced by ca. $55 \%$ compared to untreated M. bovis BCG assayed in the presence of collagen (Supplementary data). This inhibitory effect of trypsin was confirmed by using $M$. bovis BCG pretreated with $1 \mu \mathrm{g} / \mathrm{mL}$ trypsin, as shown by the complete abolition of the collagen-induced stimulation of adherence onto A549 pneumocytes and U937 macrophages. This observation suggests that $M$. bovis BCG surface proteins are involved in the collagen-dependent cytoadherence.

LBP/Hlp binds immobilized collagen I - In order to isolate the M. bovis BCG compounds that bind collagen I, exponentially-growing mycobacteria were lyzed by sonication and centrifuged. Then, the centrifugation supernatant corresponding to the soluble material was collected and directly chromatographed onto a collagen I-Sepharose matrix equilibrated in PBS. Fractions corresponding to the eluted material were analyzed by SDS-PAGE and immunoblotting (Fig. 4A). Coomassie blue staining of the polyacrylamide gel revealed the presence of a protein exhibiting an apparent molecular weight of ca. $32 \mathrm{kDa}$. The gel band containing this protein was excised and submitted to trypsin digestion. Mass spectrometry analysis of the resulting peptides revealed peptide masses corresponding to the previously described mycobacterial LBP/Hlp. To confirm the collagen-binding capacity of BCG LBP/Hlp, the recombinant protein was successfully obtained (Supplementary data) and shown in microplate binding-assays to interact with collagen I (Fig. 4B). Finally, we cultivated M. smegmatis wt and $\Delta \mathrm{lbp} / h l p$ strains in $7 \mathrm{H} 9$ medium in the absence or presence of collagen I at the concentration of $25 \mu \mathrm{g} /$ $\mathrm{mL}$. When cultures reached an $\mathrm{OD}_{600 \mathrm{~nm}}$ of 1.8 , bacteria were fixed, acid-fast stained and visualised under light microscope. Fig. 4C shows that, as expected, the mutant strain was unable to form large clumps, in contrast to the wt strain, indicating a decrease capacity to interact with collagen fibres when compared with the wt strain. These results confirmed the direct interaction between the mycobacterial LBP/Hlp and collagen.

Recombinant M. leprae LBP/Hlp binds collagen M. leprae LBP/Hlp shares $84 \%$ sequence identity with the BCG homologue and it has been characterized as an important adhesin mediating bacterial interaction with host cells (Shimoji et al. 1999, Marques et al. 2000, Lima et al. 2005, Portugal et al. 2008). Next, we then tested the capacity of recombinant $M$. leprae LBP/Hlp to bind collagen in a solid phase interaction assay. For this purpose, microplate wells were coated with M. leprae $\mathrm{rLBP} / \mathrm{Hlp}$ and following a blocking step with BSA, increasing concentrations of biotinylated collagen I were added to the wells. The complexes were finally developed using a streptavidin-peroxidase conjugate. As shown in Fig. 5A, BSA displayed no significant collagen-binding activity for collagen I. In contrast, $M$. leprae $\mathrm{rLBP} / \mathrm{Hl}$ p was demonstrated to bind collagen I in a dose-dependent and saturable manner.

Recombinant LBP/Hlp binds different collagen types via its $C$-terminal domain - The $\mathrm{NaCl}$ elution of $M$. bovis BCG LBP/Hlp from a chromatographic matrix bearing 
covalently immobilized collagen I suggested that ionic interactions were more likely involved in the adhesin interaction with collagen. The $\mathrm{C}$-terminal half of $\mathrm{LBP} / \mathrm{Hlp}$ is highly positively charged and exhibits homology with the eukaryotic class H1 histones (Lee et al. 1998, Prabhakar et al. 1998). In a recent investigation, we have used truncated recombinant $M$. leprae $\mathrm{LBP} / \mathrm{Hlp}$ molecules corresponding to the $\mathrm{N}$-terminal (residues $1-110, \mathrm{rLBP} / \mathrm{Hlp}-\mathrm{N}$ ) or the C-terminal (residues 111-200, rLBP/Hlp-C) domains and showed that the $\mathrm{C}$-terminal half of the protein constitutes the major laminin and heparin-binding site of the adhesin (Lima et al. 2005, Portugal et al. 2008). We then tested if the C-terminal domain of LBP/Hlp was the collagen-interacting site of the protein. For this purpose, we assayed the collagen-binding activity of the $M$. leprae LBP/Hlp truncated forms which were previously shown to coat microplate wells with identical efficiencies (Lima et al. 2005). Fig. 5A shows overlapping of the collagen Ibinding curves obtained with rLBP/Hlp and rLBP/Hlp-C, while no collagen I-binding activity was observed using $\mathrm{rLBP} / \mathrm{Hlp}-\mathrm{N}$. This finding indicates that the interaction of LBP/Hlp with collagen I is mediated by the C-terminal half of the adhesin. In contrast, rLBP/Hlp-N displayed very limited binding capacity to collagen I. Finally, we next tested the capacity of full-length rLBP/Hlp and truncated forms to bind to other collagen types. As shown in Fig. 5B, the rLBP/Hlp and $\mathrm{rLBP} / \mathrm{Hlp}-\mathrm{C}$ were also able to significantly bind collagens III, IV and VI. These results
A

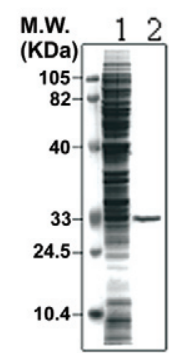

C

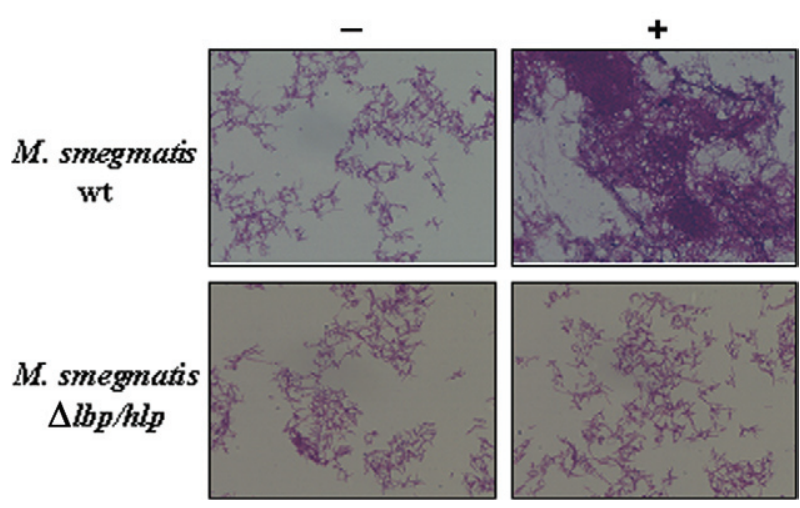

Fig. 4A: sodium dodecyl sulphate polyacrylamide gel electrophoresis and immunoblotting analyses of Mycobacterium bovis BCG collagenbinding protein. The Coomassie-blue staining of a polyacrylamide gel containing a clarified lysate of $M$. bovis BCG prior application onto a collagen-Sepharose matrix (Lane 1) and the salt-eluted material (Lane 2); B: recombinant $M$. bovis BCG laminin-binding histone-like protein (LBP/Hlp) binds collagen. Microplate wells coated with $1 \mu \mathrm{g} / \mathrm{mL}$ of collagen I were incubated with increasing concentrations of $M$. bovis BCG recombinant-LBP/Hlp. The wells were then incubated with the anti-LBP/Hlp monoclonal antibody 5G9 and, finally, with a rabbit anti-mouse IgG peroxidase conjugate; C: the Mycobacterium smegmatis wild-type (wt), but not the $\Delta h l p$ strain, was able to form large clumps in the presence of collagen I. M. smegmatis wt and the mutant for the $h l p$ gene $(\Delta h l p)$ were grown in complete Middlebrook $7 \mathrm{H} 9$ broth supplemented or not with type 1 calf skin collagen at the concentration of $25 \mu \mathrm{g} / \mathrm{mL}$. When cultures reached an $\mathrm{OD}_{600 \mathrm{~mm}}$ of 1.8 , mycobacteria were acid-fast stained by Kynion and visualized under light microscope with 400X magnification; BSA: bovine serum albumin.
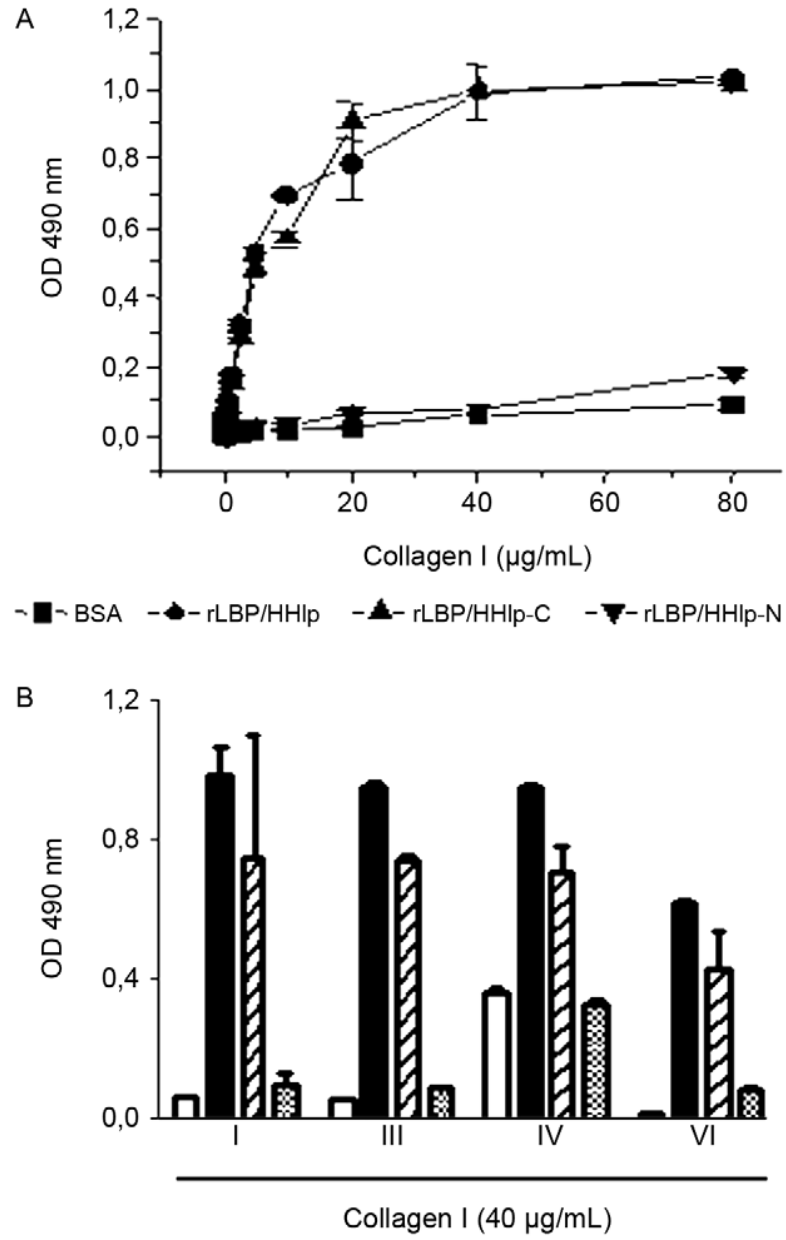

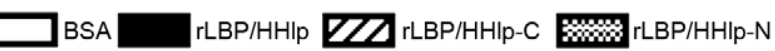

Fig. 5: Mycobacterium leprae laminin-binding histone-like protein (LBP/Hlp) binds to different collagen types by its C-terminal domain. Microplate wells coated with $5 \mu \mathrm{g} / \mathrm{mL}$ of full-length $M$. leprae recombinant-LBP/Hlp, truncated N-terminal (rLBP/Hlp-N) or C-terminal (rLBP/Hlp-C) domains were incubated with increasing concentrations of biotinylated collagen I (A) or $40 \mu \mathrm{g} / \mathrm{mL}$ of collagens I, III, IV or VI (B). Binding is expressed in absorbency units at $490 \mathrm{~nm}$. Data represent the mean $+/$ - standard deviation of a typical experiment done in duplicate. Five experiments were performed with similar results. BSA: bovine serum albumin. 
suggest that, similarly to its interaction with heparin and laminin, LBP/Hlp binds collagen mainly through interactions involving the lysine-rich repeats present in the C-terminal domain of the adhesin.

\section{DISCUSSION}

Because specific interactions with host cells represent a crucial and early step in any infectious process, pathogenic microorganisms have evolved adhesins capable to bind membrane receptors or compounds present in the eukaryotic extracellular matrices (Westerlund \& Korhonen 1993). Pathogenic mycobacteria synthesize a wide panel of adhesins involved in the bacterial interaction with epithelial cells and/or monocyte-derived professional phagocytes (Hoppe et al. 1997, Sidobre et al. 2000, Diaz-Silvestre et al. 2005, Kinhikar et al. 2006, Ragas et al. 2007, Hickey et al. 2010). Several proteinaceous mycobacterial adhesins have been demonstrated to recognize mammalian extracellular matrix components. Such an adhesive activity has been demonstrated for the proteins belonging to the antigen 85 complex and the fibronectin attachment protein, which both bind fibronectin (Abou-Zeid et al. 1991, Peake et al. 1993, Zhao et al. 1999), the HBHA which interacts with heparan sulphate-containing proteoglycans (Delogu \& Brennan 1999, Pethe et al. 2002) and the LBP/Hlp (also called MDP1) that binds laminin, heparan sulphate chains and HA (Shimoji et al. 1999, Aoki et al. 2004, Lima et al. 2005, Lefrançois et al. 2011).

Evidence strongly suggests that LBP/Hlp displays a bi-functional role in the biology of mycobacteria, acting both in nuclei acid metabolism (Prabhakar et al. 1998) and as an adhesin. In this regard, LBP/Hlp was initially characterized as a LBP, mediating the binding and invasion of SC by M. leprae (Shimoji et al. 1999, Marques et al. 2000). Recombinant LBP/Hlp was able to avidly bind laminin in solid phase assays and the exogenous addition of the protein significantly enhances bacterial binding to SC (Marques et al. 2000). These data, together with the recent finding indicating that $M$. leprae actively expresses LBP/Hlp during the human infection (Lima et al. 2005), have reinforced the involvement of LBP/Hlp in the successful invasion and colonization of the peripheral nerve system by M. leprae. More recently, LBP/Hlp was also shown to play an important role in mycobacteria-lung epithelial cell interaction. Both M. tuberculosis and M. bovis BCG were shown to bind and invade A549 human lung epithelial cells through the interaction of LBP/Hlp with HA presence on the cell surface (Aoki et al. 2004).

Collagenous proteins, which are predominant constituents of the mammalian extracellular matrix, can also function as receptors for the adhesion of microbial pathogens. The collagen-binding microbial surface components recognizing adhesive matrix molecules (MSCRAMMs) that are synthesized by both Gram-positive and Gram-negative bacteria constitute a large family of proteins involved in the bacterial attachment to host tissues (Patti et al. 1994). Therefore, it is not surprising that collagen-binding MSCRAMMs may represent important virulence factors such as the CNA, YadA, Cpa and Dr adhesins, which are produced by Staphylococ- cus aureus, Yersinia enterocolitica, Streptococcus pyogenes and Escherichia coli, respectively and which play a role in the establishment and progression and/or persistence of the infection (Gripenberg-Lerche et al. 1994, Selvarangan et al. 2004, Xu et al. 2004, Kreikemeyer et al. 2005). Besides adhesins that bind collagen, more recently bacterial collagen-like surface proteins have been characterized and shown to play a role in microbial pathogenesis by mediating attachment to host cell collagen receptors (Caswell et al. 2008). So far, no studies have reported the interaction of mycobacteria with collagen. Here, we show for the first time that mycobacteria may interact directly with collagen proteins via the surface-exposed LBP/Hlp adhesin.

Evidence that mycobacteria can interact with collagen was initially obtained from the observation that collagen I was able to induce aggregation of bacterial cells. Images generated by SEM also showed collagen fibres interacting with the surface of bacterial cell. This interaction was confirmed by the observation that exogenous collagen was able to increase mycobacterial adherence to human type II A549 pneumocytes and U937 human macrophages in a saturable and dose-dependent manner. An affinity chromatography performed on collagen I-Sepharose allowed the purification of a collagen-binding component from BCG cell lysate that was identified by mass spectrometry as the LBP/Hlp protein. Furthermore, when produced in the recombinant form, $\mathrm{BCG} \mathrm{LBP} / \mathrm{Hlp}$ protein was able to bind collagen in microplate solid phase assays confirming that $\mathrm{LBP} / \mathrm{Hlp}$ is also a collagen-binding protein. $\mathrm{LBP} / \mathrm{Hlp}$ is a highly conserved protein detected on the surface of all mycobacterial species so far investigated (Lefrançois et al. 2011). To show that LBP/Hlp is the major collagenbinding protein present on mycobacterial surface, we analyzed the interaction of collagen fibres with $M$. smegmatis $\Delta h l p$, the only hlp null mutant so far available in the context of mycobacterial species. This mutant has been characterized and used in several previous studies (Lee et al. 1998, Marques et al. 2000, Biet et al. 2007). In contrast to the wt strain, $M$. smegmatis $\Delta h l p$ was unable to interact and form large clumps when grown in the presence of collagen I, suggesting that LBP/Hlp represents a major component on bacterial surface mediating microbial interaction with collagen.

Mycobacterial LBP/Hlp is about twice the size of other bacterial Hlps. The C-terminal half of mycobacterial Hlp is absent from most other bacteria and has an unusual amino acid composition owing to a high alanine, lysine and proline content, which resembles the C-terminal region of eukaryotic class H1 histones (Prabhakar et al. 1998). This highly positive domain was recently shown to mediate the interaction of LBP/Hlp with laminin and heparan sulphate and also with SC (Lima et al. 2005). To map the region of LBP/Hlp responsible for the collagen binding activity, truncated versions of the adhesin corresponding to the $\mathrm{N}$-terminal and $\mathrm{C}$-terminal half of the protein were generated and tested in a solid phase collagen binding assay. These experiments clearly demonstrated that the lysine-rich C-terminal region holds most if not all of the collagen-binding properties of the protein. Both full-length protein and the C-terminal domain were 
able to bind very similarly to collagen type I, while interaction with collagen III, IV and VI was partially mediated by the $\mathrm{C}$-terminal domain. These data reinforce the idea that LBP/Hlp C-terminal domain is responsible for most if not all of the adhesive property of this protein.

The definition of the molecules used by M. tuberculosis and $M$. leprae to gain access and colonize different tissues of the human body may provide clues for the development of new therapeutic and/or prophylactic strategies to better control tuberculosis and leprosy. The capacity of LBP/Hlp to bind different collagen types here demonstrated reinforces its potential role as a virulence factor of pathogenic mycobacteria. The binding properties of LBP/ Hlp to multiple extracellular matrix components suggest that this protein may play an important role in distinct moments during the natural course of human infection. As previously suggested, early during infection, LBP/ Hlp might play, in conjunction with HBHA, a key role on bacterial attachment to respiratory epithelial cells (Aoki et al. 2004). We can also speculate on the involvement of LBP/Hlp in bacterial dissemination inside the human body that occurs both in leprosy and tuberculosis (Pessolani et al. 2003). During this process, the bacilli might need to interact with distinct basal membranes, which are essentially composed of laminin and collagen IV (Kreis \& Vale 1993). Finally, LBP/Hlp may also participate of the colonization of vulnerable tissues such as the peripheral nerve system by $M$. leprae through interaction mainly with laminin (Shimoji et al. 1999, Marques et al. 2000) and the bones and joints by M. tuberculosis, which are rich in collagen I (Kreis \& Vale 1993).

\section{ACKNOWLEDGEMENTS}

To Dr John S Spencer, from the Colorado State University, for providing the $5 \mathrm{G} 9$ anti-rLBP/Hlp monoclonal antibody, to Elizabeth Pradel and Yves Dufrêne, for critical reading of the manuscript, to Jean-Pierre Tissier, for the technical assistance, and to the memory of Franco Dante Menozzi, who died in July 2005.

\section{REFERENCES}

Abou-Zeid C, Garbe T, Lathigra R, Wiker HG, Harboe M, Rook GA, Young DB 1991. Genetic and immunological analysis of Mycobacterium tuberculosis fibronectin-binding proteins. Infect Immun 59: 2712-2718.

Aoki K, Matsumoto S, Hirayama Y, Wada T, Ozeki Y, Niki M, Domenech P, Umemori K, Yamamoto S, Mineda A, Matsumoto M, Kobayashi K 2004. Extracellular mycobacterial DNA-binding protein 1 participates in mycobacterium-lung epithelial cell interaction through hyaluronic acid. J Biol Chem 279: 39798-39806.

Biet F, Marques MAM, Grayon M, Xavier da Silveira EK, Brennan PJ, Drobecq H, Raze D, Vidal Pessolani MC, Locht C, Menozzi FD 2007. Mycobacterium smegmatis produces an HBHA homologue which is not involved in epithelial adherence. Microbes Infect 9: 175-182.

Caswell CC, Barczyk M, Keene DR, Lukomska E, Gullberg DE, Lukomski $S$ 2008. Identification of the first prokaryotic collagen sequence motif that mediates binding to human collagen receptors, integrins alpha2beta1 and alpha11beta1. J Biol Chem 283: 36168-36175.

Delogu G, Brennan MJ 1999. Functional domains present in the mycobacterial hemagglutinin, HBHA. J Bacteriol 181: 7464-7469.
Diaz-Silvestre H, Espinosa-Cueto P, Sanchez-Gonzalez A, EsparzaCeron MA, Pereira-Suarez AL, Bernal-Fernandez G, Espitia C, Mancilla R 2005. The 19-kDa antigen of Mycobacterium tuberculosis is a major adhesin that binds the mannose receptor of THP-1 monocytic cells and promotes phagocytosis of mycobacteria. Microb Pathog 39: 97-107.

Dupres V, Menozzi FD, Locht C, Clare BH, Abbott NL, Cuenot S, Bompard C, Raze D, Dufrêne YF 2005. Nanoscale mapping and functional analysis of individual adhesins on living bacteria. Nat Methods 2: 515-520.

Ernst JD 1998. Macrophage receptors for Mycobacterium tuberculosis. Infect Immun 66: 1277-1281.

Gripenberg-Lerche C, Skurnik M, Zhang L, Söderström KO, Toivanen P 1994. Role of YadA in arthritogenicity of Yersinia enterocolitica serotype O:8: experimental studies with rats. Infect Immun 62: 5568-5575.

Hickey TB, Ziltener HJ, Speert DP, Stokes RW 2010. Mycobacterium tuberculosis employs Cpn60.2 as an adhesin that binds CD43 on the macrophage surface. Cell Microbiol 12: 1634-1647.

Hoppe HC, de Wet BJ, Cywes C, Daffé M, Ehlers MR 1997. Identification of phosphatidylinositol mannoside as a mycobacterial adhesin mediating both direct and opsonic binding to nonphagocytic mammalian cells. Infect Immun 65: 3896-3905.

Kinhikar AG, Vargas D, Li H, Mahaffey SB, Hinds L, Belisle JT, Laal S 2006. Mycobacterium tuberculosis malate synthase is a laminin-binding adhesin. Mol Microbiol 60: 999-1013.

Kreikemeyer B, Nakata M, Oehmcke S, Gschwendtner C, Normann J, Podbielski A 2005. Streptococcus pyogenes collagen type Ibinding Cpa surface protein. Expression profile, binding characteristics, biological functions and potential clinical impact. $J$ Biol Chem 280: 33228-33239.

Kreis T, Vale R 1993. Guidebook to the extracellular matrix and adhesion proteins, Oxford University Press, Oxford, 176 pp.

Lee BH, Murugasu-Oei B, Dick T 1998. Upregulation of a histonelike protein in dormant Mycobacterium smegmatis. Mol Gen Genet 260: 475-479.

Lefrançois LH, Pujol C, Bodier CC, Teixeira-Gomez AP, Drobecq H, Rosso ML, Raze D, Dias AA, Hugot JP, Chacon O, Barletta RG, Locht C, Pessolani MCV, Biet F 2011. Characterization of the Mycobacterium avium subsp. paratuberculosis laminin-binding/ histone-like protein ( $\mathrm{Lbp} / \mathrm{Hlp})$ which reacts with sera from patients with Crohn's disease. Microbes Infect 13: 585-594.

Lima CS, Marques MA, Debrie AS, Almeida EC, Silva CA, Brennan PJ, Sarno EN, Menozzi FD, Pessolani MC 2009. Heparin-binding hemagglutinin (HBHA) of Mycobacterium leprae is expressed during infection and enhances bacterial adherence to epithelial cells. FEMS Microbiol Lett 292: 162-169.

Lima CS, Zulianello L, Marques MA, Kim H, Portugal MI, Antunes SL, Menozzi FD, Ottenhoff TH, Brennan PJ, Pessolani MC 2005. Mapping the laminin-binding and adhesive domain of the cell surface-associated Hlp/LBP protein from Mycobacterium leprae. Microbes Infect 7: 1097-1099.

Marques MAM, Mahapatra S, Nandan D, Dick T, Sarno EN, Brennan PJ, Pessolani MCV 2000. Bacterial and host-derived cationic proteins bind alpha2-laminins and enhance Mycobacterium leprae attachment to human Schwann cells. Microbes Infect 2: 1407-1417.

Menozzi FD, Reddy VM, Cayet D, Raze D, Debrie AS, Dehouck MP, Cecchelli R, Locht C 2006. Mycobacterium tuberculosis heparin-binding haemagglutinin adhesin (HBHA) triggers receptormediated transcytosis without altering the integrity of tight junctions. Microbes Infect 8: 1-9. 
Menozzi FD, Rouse JH, Alavi M, Laude-Sharp M, Muller J, Bischoff $\mathrm{R}$, Brennan MJ, Locht C 1996. Identification of a heparin-binding hemagglutinin present in mycobacteria. J Exp Med 184: 993-1001.

Mueller-Ortiz SL, Sepulveda E, Olsen MR, Jagannath C, Wanger AR, Norris SJ 2002. Decreased infectivity despite unaltered C3 binding by a DeltahbhA mutant of Mycobacterium tuberculosis. Infect Immun 70: 6751-3760.

Patti JM, Allen BL, McGavin MJ, Höök M 1994. MSCRAMM-mediated adherence of microorganisms to host tissues. Annu Rev Microbiol 48: 585-617.

Peake P, Gooley A, Britton WJ 1993. Mechanism of interaction of the 85B secreted protein of Mycobacterium bovis with fibronectin. Infect Immun 61: 4828-4834.

Pessolani MCV, Marques MA, Reddy VM, Locht C, Menozzi FD 2003. Systemic dissemination in tuberculosis and leprosy: do mycobacterial adhesins play a role? Microbes Infect 5: 677-684.

Pethe K, Alonso S, Biet F, Delogu G, Brennan MJ, Locht C, Menozzi FD 2001. The heparin-binding haemagglutinin of M. tuberculosis is required for extrapulmonary dissemination. Nature 412: 190-194.

Pethe K, Aumercier M, Fort E, Gatot C, Locht C, Menozzi FD 2000. Characterization of the heparin-binding site of the mycobacterial heparin-binding hemagglutinin adhesin. $J$ Biol Chem 275: 14273-14280.

Pethe K, Bifani P, Drobecq H, Sergheraert C, Debrie AS, Locht C, Menozzi FD 2002. Mycobacterial heparin-binding hemagglutinin and laminin-binding protein share antigenic methyllysines that confer resistance to proteolysis. Proc Natl Acad Sci USA 99: 10759-10764.

Portugal MI, Todeschini AR, de Lima CS, Silva CA, Mohana-Borges R, Ottenhoff TH, Mendonça-Previato L, Previato JO, Pessolani MC 2008. Characterization of two heparin sulphate-binding sites in the mycobacterial adhesin Hlp. BMC Microbiol 8: 75.

Prabhakar S, Annapurna PS, Jain NK, Dey AB, Tyagi JS, Prasad HK 1998. Identification of an immunogenic histone-like protein (HLPMt) of Mycobacterium tuberculosis. Tuber Lung Dis 79: 43-53.
Ragas A, Roussel L, Puzo G, Rivière M 2007. The Mycobacterium tuberculosis cell-surface glycoprotein apa as a potential adhesin to colonize target cells via the innate immune system pulmonary C-type lectin surfactant protein A. J Biol Chem 282: 5133-5142.

Selvarangan R, Goluszko P, Singhal J, Carnoy C, Moseley S, Hudson B, Nowicki S, Nowicki B 2004. Interaction of Dr adhesin with collagen type IV is a critical step in Escherichia coli renal persistence. Infect Immun 72: 4827-4835.

Shimoji Y, Ng V, Matsumura K, Fischetti VA, Rambukkana A 1999. A 21-kDa surface protein of Mycobacterium leprae binds peripheral nerve laminin-2 and mediates Schwann cell invasion. Proc Natl Acad Sci USA 96: 9857-9862.

Sidobre S, Nigou J, Puzo G, Rivière M 2000. Lipoglycans are putative ligands for the human pulmonary surfactant protein $\mathrm{A}$ attachment to mycobacteria. Critical role of the lipids for lectin-carbohydrate recognition. J Biol Chem 275: 2415-2422.

Stokes RW, Haidl ID, Jefferies WA, Speert DP 1993. Mycobacteriamacrophage interactions. Macrophage phenotype determines the nonopsonic binding of Mycobacterium tuberculosis to murine macrophages. J Immunol 151: 7067-7076.

Westerlund B, Korhonen TK 1993. Bacterial proteins binding to the mammalian extracellular matrix. Mol Microbiol 9: 687-694.

WHO - World Health Organization 2010a. Global leprosy situation 2010. Wkly Epidemio Rec 85: 337-334.

WHO - World Health Organization 2010b. Global tuberculosis control 2010. Wkly Epidemio Rec 85: 69-80.

Xu Y, Rivas JM, Brown EL, Liang X, Höök M 2004. Virulence potential of the staphylococcal adhesin CNA in experimental arthritis is determined by its affinity for collagen. J Infect Dis 189 : 2323-2333.

Zhao W, Schorey JS, Groger R, Allen PM, Brown EJ, Ratliff TL 1999. Characterization of the fibronectin binding motif for a unique mycobacterial fibronectin attachment protein, FAP. J Biol Chem 274: 4521-4526. 
A

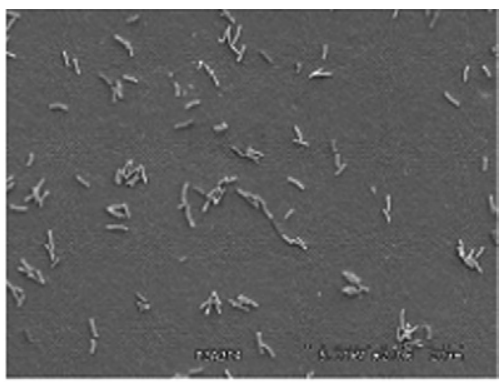

B

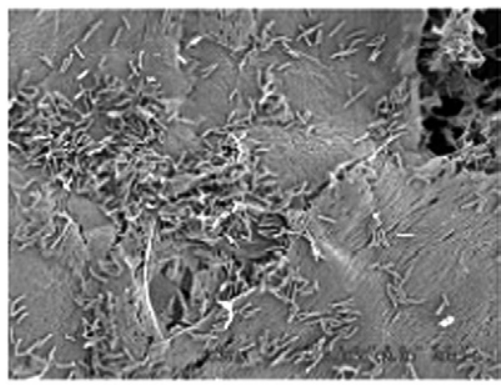

BCG

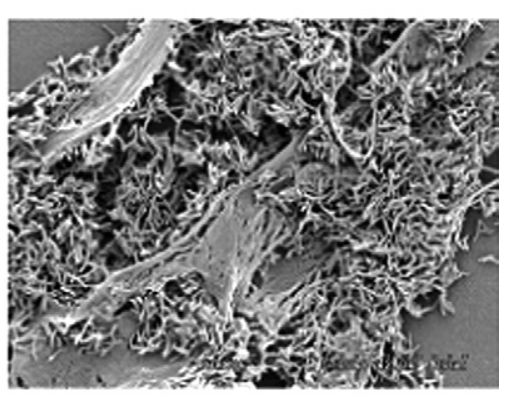

C

collagen I

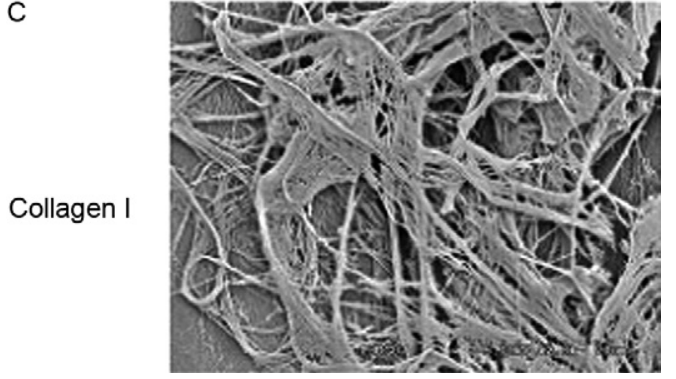

Scanning electron micrographs of Mycobacterium bovis BCG grown in the absence or the presence of collagen I. Mycobacteria grown in Sauton medium (Panel A) or in Sauton medium supplemented with $25 \mu \mathrm{g} / \mathrm{mL}$ collagen I (Panel B) were resuspended in phosphate buffered saline (PBS) and fixed for scanning electron microscopy (SEM) examination. A similar fixation step was applied to a collagen I solution prepared in PBS, followed by SEM observation (Panel C). 
U937 macrophages

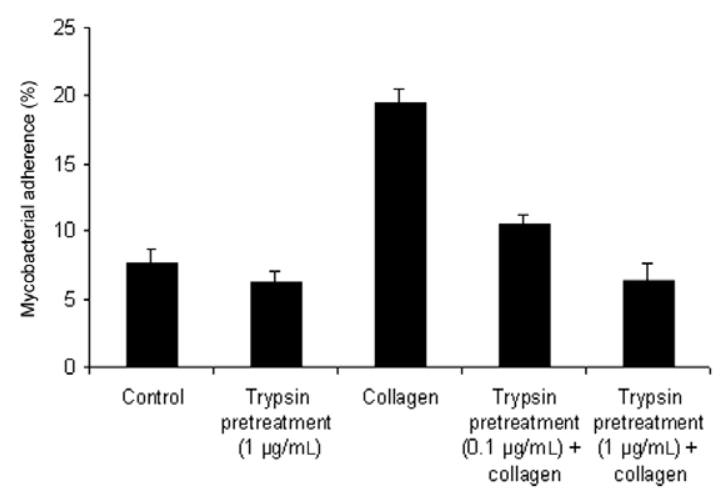

A549 pneumocytes

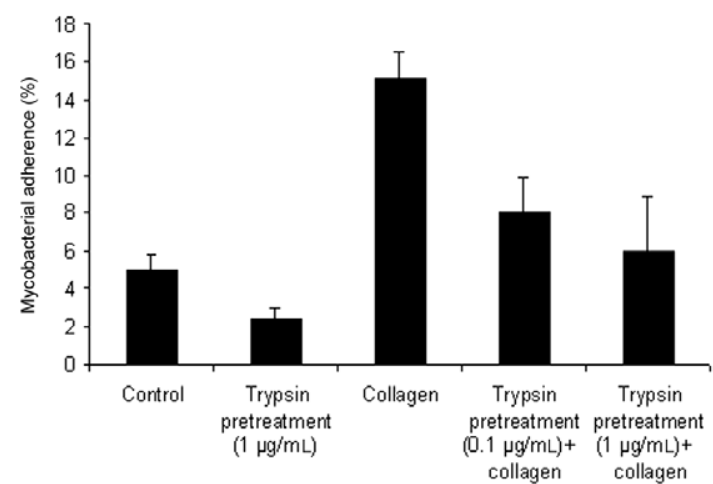

Effect of trypsin pretreatment on the Mycobacterium bovis BCG cytoadherence. Exponentially-growing mycobacteria were pretreated with $0.1 \mu \mathrm{g} / \mathrm{mL}$ or $1 \mu \mathrm{g} / \mathrm{mL}$ trypsin and then assayed for their interaction with A549 pneumocytes or U937 macrophages (multiplicity of infection of 10) in the absence or presence of $50 \mu \mathrm{g} / \mathrm{mL}$ exogenous collagen I. Colony forming units associated with target cells were counted by plating serial cell lysate dilutions on 7H11 agar. The data with standard deviations represent averages for quadruplicate experiments.

A

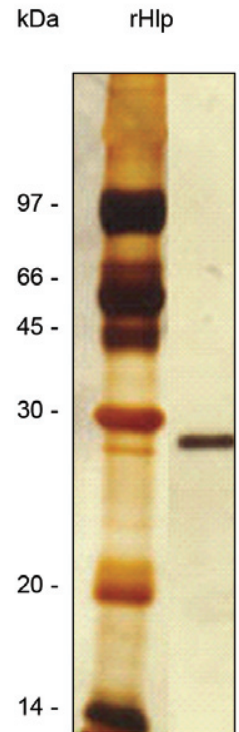

$\mathrm{kDa} \quad \mathrm{rHlp}$

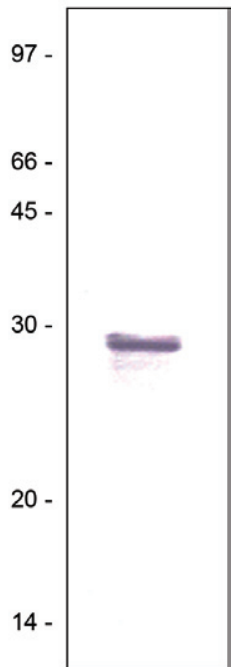

Purity and integrity of the BCG recombinant-laminin-binding histone-like protein (LBP/Hlp). Purified BCG rLBP/Hlp was subjected to $15 \%$ sodium dodecyl sulphate polyacrylamide gel electrophoresis. A: gel stained with silver; B: immunoblotting developed with anti-LBP/Hlp (5G9) monoclonal antibody. The molecular weight markers are indicated on the left. 